\title{
Rapid Determination of Free Prolyl Dipeptides and 4- hydroxyproline in Urine Using Flow-gated Capillary Electrophoresis
}

\author{
Ning Zhang \\ Faculty: Maojun Gong \\ Department of Chemistry, Fairmount College of Liberal Arts and Sciences
}

4-hydroxyproline (Hyp) and prolyl dipeptides such as prolyl hydroxyproline (Pro-Hyp) have been found as disease biomarkers in the diagnosis of bone turnover and osteoporosis. To determine these compounds in human urine samples, we report a rapid and accurate method for the selective detection of free prolyl dipeptides and 4-hydroxyproline (secondary amines) by using flow-gated capillary electrophoresis coupled with laser-induced fluorescence (CE-LIF) detection method. To do that, primary amines in urine samples were first converted to tertiary amines thus got blocked with o-phthalaldehyde, and then the secondary amines were derivatized selectively by using 4-fluoro-7-nitro-2,1,3-benzoxadiazole (NBD-F) at room temperature. The derivatized mixture was analyzed and quantified by a flow-gated CE-LIF system. Analytical conditions were optimized to have the background electrolyte (BGE) solution composed of tetraborate ( $\mathrm{pH}$ 9.2), cholate and deoxycholate at $40 \mathrm{mM}$ each, respectively. We were able to separate and detect six prolyl compounds, namely Pro-Hyp, Pro-Pro, Pro-Gly, Pro-Leu, Hyp and Pro in unhydrolyzed urine samples, and the overall separation time (30 s) was 60 folds shorter than the reported HPLC method. The detection limits (LOD) were around $20 \mathrm{nM}$ for dipeptides, and were around $60 \mathrm{nM}$ for Hyp and Pro. The concentrations of these prolyl compounds in fresh urine samples were determined by using one-point standard addition method for which nipecotic acid was chosen as the internal standard. Compared with other reported methods, this technique was not only significantly simplified, but it could also greatly shorten the separation time while ensuring the accuracy of measurements. This method can also be applied to detecting prolyl dipeptides and Hyp in other biological fluids such as blood plasma. 